\title{
Micronutrients Delivery: The Unwritten Conundrum
}

\section{Kok-Ping Chan}

Institute of Chemical and Engineering Science, 8 Biomedical Grove, Neuros, 07-01, Singapore 138665

Micronutrient deficiency is one of the most pressing worldwide concerns from the lens of public health and socioeconomic standpoint. The World Health Organization (WHO) estimated that more than 2 billion people worldwide suffer from vitamin and mineral deficiencies, primarily iodine, iron, vitamin A and zinc, with important health consequences.1An understanding of micronutrients uptake and metabolism of micronutrients is a key research interest amongst both academics and industry. However, scientific and clinical investigation of this perplexing topic is often a complex endeavor, giving rise to slow advancement in the efforts to enhance nutritional uptake in functional food. Although there is already a rich knowledge of the nutritional benefits of the various macro- and micro-nutrients, the question is how efficient is the uptake of these compounds in the body? Indeed, the daily required intake is for micronutrients is usually very minute, the underlying challenge is in the relative abundance of these compounds, as well as their bioavailability in the human gut.

Many consumers often resort to taking health supplements to fortify themselves with the necessary micronutrients. Because of this,companies pour in huge investments to research on nutritional supplements, making quantumleapsin the technologicaladvancements that lead to numerous products in the current market. The truth is,many of these products are designed to reach out to the affluent class of people, who probably already have sufficient intake from the diet. The greater problem is widespread consumption of poor-quality food leading to "hidden hunger", which ravages economies and worsens poverty.The challenge now is how to incorporate micronutrients into staple food, without compromising stability, savory, and most importantly, toenhancedelivery in the human metabolic system.

Formulation of functional food is a sustainable way to address the"hidden hunger" issue. The current concept of micro-nutrients fortification is overdosing with key ingredients more than the daily requirement. For examplein a typical $0.5 \mathrm{~g}$ tablet for zinc fortification, the zinc content is only about $15-50 \mathrm{mg}$, which is already many times more than the daily required intake. The rest of the tablet is made up of excipients such as maltodextrin, cellulose, silica, and lubricants such as stearates and fatty acids. The formulation of nutritional supplements varies, depending on the type, combination and purpose of the micronutrients required. However, one fact remains unchanged - the bulk of the pill comprises of components that the body do not necessarily require, but will still add on to the body's metabolic burden.

The manufacturing of health supplements is very similar to the production of active pharmaceutical ingredients, where strict regulatory guidelines have to be adhered and intensive manufacturing infrastructures needs to be in place. Such technology centric activity not only draws away valuable resources from the more important food manufacturing industry, but also at times,provided misleadinginformation dietary supplements to the consumers. Research2has shown that the bioavailability of supplementary micronutrients is limited, suggesting that much of the key ingredient in the formulation is typically unabsorbed by the human body. Hence, from the angle of engineering and economic assessment, the strategy to combat global micronutrients deficiency through supplements is not a sustainable one.

\section{Publication History:}

Received: December 21, 2015

Accepted: December 27, 2015

Published: December 29, 2015

There are two ways we can fortify micronutrients in food systems. Firstly, by genetic engineering where crops can be evolved to have enhanced levels of the required micronutrients. There is an increasing trend in focusing research on genetic evolution of crops to produce higher levels of vitamins and biomolecules such as biotin. However, these endeavors possess inherent scientific challenges. The biosynthesis machineries are not designed to produce these compounds in quantities more than what is necessary for the plants' survival. There is a very delicate balance in the nature's way of producing useful micronutrients, by which a deliberate attempt to deviate from track can cause enormous impacts on the symbiotic ecological system. On the other hand, genetic engineering can only address deficiencies in micronutrients that are organic based, such as vitamins $\mathrm{A}$ to $\mathrm{K}$. Other essential minerals such as iron, zinc cannot be biosynthesized, and can only be fortified through a more efficient uptake and retention by the plant system. Such challenge can only be addressed by a convergence of efforts to spearhead plant biology research with respect to agricultural applications. The "Golden Rice Project" is on example of such endeavor.3,4However, even with the successes in achieving genetically fortified micronutrients in crops, the next challenge is how to ensure efficient delivery of these compounds in the human body.

Secondly, by food processing where micronutrients can be encapsulated within the food matrix itself, such as bread, noodles. The rationale is simple. Incorporation or fortification of essential micronutrients in staple food provides an equalized platform for people to attain the required dietary intake based on body mass. As developed nations promised more available staple food for people in the developing counterparts, the macro-nutritional aspect has been well taken care of in terms of combatting hunger. However, the micro-nutritional part is often overlooked. Hence fortification of vitamins and minerals become an important tool to address the issue of hidden hunger.Fortification by merely increasing the amount of micronutrients in food only serves to scratch the surface of the problem. The real challenge is how to deliver the key ingredients to the right place, in the right amount at the right time. Encapsulation technologies are potential solutions to effective micronutrients delivery in the gut.

So, how does encapsulation actually work? In simple terms, encapsulation is encasing active ingredient in suitable matrix to either enhance its stability, protects it from harsh environment or enables

"Corresponding Author: Dr. Kok-Ping Chan, Institute of Chemical and Engineering Science, 8 Biomedical Grove, Neuros, 07-01, Singapore 138665; E-mail: chan_kok_ping@ices.a-star.edu.sg

Citation: Chan KP (2016) Micronutrients Delivery: The Unwritten Conundrum. Int J Clin Nutr Diet 1: 105. doi: http://dx.doi.org/10.15344/ijcnd/2015/105

Copyright: () 2016 Chan. This is an open-access article distributed under the terms of the Creative Commons Attribution License, which permits unrestricted use, distribution, and reproduction in any medium, provided the original author and source are credited. 
its release to perform specific biological function(s).5However, complexities in the choice of excipients, processing parameters, nature of the active micronutrients, the specific delivering requirements areall important considerations in determining the efficiency and effectiveness of encapsulation. Hence, there is an emerging trend to develop new encapsulation technologies that lead to new frontiers in nutrition delivery. Encapsulation can provide a mechanism to enhance the stability of sensitive micronutrients by exclusion of air, water, light and heat. For example, iron is prone to oxidation in nature state, formingiron (III) complexes which do not have nutritional benefits. For organic compounds such as vitamin A, which possess olefin functional groups that are also susceptible to redox reactions, encapsulation is a promising method to incorporate micronutrients into functional food systems that can tolerate harsh food processing conditions, and enable efficient delivery into the gut without compromising the integrity of the nutrients.

Encapsulation technologies for living organisms such as probiotics have also emerged as a new area in functional food research. The paradox here is that while scientists can exploit on the most resistant materials for encapsulation, there must be consideration for the release of the micro-nutrients upon appropriate triggers. For example, probiotics needs to be untouched from the bucal cavity all the way to its release in the gut. In this food passage, the encapsulant has to be able to resist mechanical disruption through mastication, transitions in $\mathrm{pH}$ and temperature to offer adequate protection to the active functional bacteria. The release of the probiotics has to be activated through an engineered mechanism that is uniquely defined. Hence the development of time-triggered capsules to encapsulate micronutrients would be an innovative strategy for such applications.

Liquid encapsulation using microemulsion is an alternative form to deliver micronutrients to the gut. Microemulsions are clear, thermodynamically stable, isotropic liquid mixtures of oil, water and surfactant, frequently in combination with a cosurfactant. Such a system is highly applicable to many organic micronutrients where the oil/water system can entrap key ingredients and release them into the system without the use of harsh conditions. The global liquid encapsulation materials market is projected to grow at a CAGR of $6.7 \%$ between 2015 and 2020, from an estimated value of USD 1,011.97 million in 2015 to reach USD 1,397.46 million by 2020 .

In summary, the challenge that lies ahead in the realm of micronutrients delivery is exciting. The realization of efficient encapsulation technologyfor micronutrients would be the silver bullet to target world-wide negative impact of micronutrients deficiencies. Through fortification into staple food matrixes, the delivery of micronutrients becomes more efficient.The science behind micronutrients deliver can only be driven by integration chemical engineering, chemistry and biology.

\section{References}

1. World Health Organization (2000) World health report Geneva WHO.

2. Sauberlich HE (1985) Bioavailability of vitamins. Prog Food Nutr Sci 9: $1-33$.

3. Ye X, Al-Babili S, Klöti A, Zhang J, Lucca P, et al. (2000) Engineering the provitaminA (beta-carotene) biosynthetic pathway into (carotenoid-free) rice endosperm. Science 287: 303.

4. Goto F, Yoshihara T, Shigemoto N, Toki S, Takaiwa F (1999) Iron fortification of rice seed by the soybean ferritin gene. Nat Biotechnol 17: 282.

5. Zimmermann MB (2004) The potential of encapsulated iron compounds in food fortification: a review. Int J VitamNutr Res. 74: 453.
11. Pimenta AM, Beunza JJ, Sanchez-Villegas A, Bes-Rastrollo M, MartinezGonzalez MA (2011) Childhood underweight, weight gain during childhood to adolescence/young adulthood and incidence of adult metabolic syndrome in the SUN (Seguimiento Universidad de Navarra) Project. Public Health Nutr 14: 1237-1244.

12. Teleman AA, de Waure C, Soffiani V, Poscia A, Di Pietro ML (2015) Physical activity and health promotion in Italian university students. Ann Ist Super Sanita 51: 106-110.

13. Hughes $V(2013)$ The big fat truth. Nature 497: 428-430.

14. Flegal KM, Kit BK, Orpana H, Graubard BI (2013) Association of all-cause mortality with overweight and obesity using standard body mass index categories: a systematic review and meta-analysis. JAMA 309: 71-82.

15. Berrington de Gonzalez A, Hartge P, Cerhan JR, Flint AJ, Hannan L, et al. (2010) Body-mass index and mortality among 1.46 million white adults. N Engl J Med 363: 2211-2219. 